\title{
A randomized trial comparing the pharmacology of magnesium sulfate when used to treat severe preeclampsia with serial intravenous boluses versus a continuous intravenous infusion
}

\author{
Thomas Easterling ${ }^{1 *}$ (D), Mary Hebert ${ }^{2}$, Hillary Bracken ${ }^{3}$, Emad Darwish ${ }^{4}$, Mohamed Cherine Ramadan ${ }^{5}$, \\ Salwa Shaarawy ${ }^{4}$, Dyanna Charles ${ }^{3}$, Tamer Abdel-Aziz $^{4}$, Ahmed Shokry Nasr $^{5}$, Sherif Mohamed Safwal ${ }^{5}$ \\ and Beverly Winikoff ${ }^{3}$
}

\begin{abstract}
Background: Magnesium sulfate is the preferred pharmacological intervention for the prevention and treatment of eclamptic seizures in pregnancy. Pain associated with intramuscular injections and the need for an electronic infusion pump for use intravenously represent significant barriers to broader utilization. We hypothesize that an alternative regimen based on serial intravenous (IV) boluses can produce serum concentrations comparable to those produced by a continuous infusion.

Methods: An open-label randomized trial was performed at two hospitals in Egypt. Women with severe preeclampsia were eligible and enrolled between January 2015 and February 2016. Two hundred subjects were randomized by random numbers generated centrally in distinct blocks and stratified by study site. They were assigned to a continuous infusion arm, (4 g loading dose with $1 \mathrm{~g} / \mathrm{hr}$. continuous infusion) or a serial IV bolus arm, (6 g loading dose with $2 \mathrm{~g}$ bolus every $2 \mathrm{~h}$ using a Springfusor ${ }^{\oplus}$ pump). Sparsely sampled magnesium serum concentrations were collected, nonlinear mixed effect modeling was conducted and Monte Carlo simulations were used to generate 200 simulated subjects in each treatment arm. The simulated populations were used to determine area under the concentration-time curve (AUC) as a measure of total drug exposure and compared.
\end{abstract}

Results: Simulated area under the magnesium serum concentration-time curve was significantly higher in the serial IV bolus arm than in the continuous infusion arm (1107 $\pm 461 \mathrm{mmol} \cdot \mathrm{min} / \mathrm{L}$ vs. $1010 \pm 398 \mathrm{mmol} \cdot \mathrm{min} / \mathrm{L},(P=0.02)$ ). Four percent of women in the serial bolus arm considered the treatment unacceptable or very unacceptable compared to $2 \%$ in the continuous infusion arm, $(P=0.68)$.

Conclusions: Serial IV boluses achieve serum magnesium concentrations statistically significantly higher but clinically comparable to those achieved with a continuous infusion and offer a third option for the administration of $\mathrm{MgSO}_{4}$ to women with preeclampsia that may reduce barriers to utilization.

Trial registration: Trial no. NCT02091401, March 17, 2014.

Keywords: Preeclampsia, Magnesium sulfate, Pharmacokinetics

\footnotetext{
* Correspondence: easter@uw.edu

'Department of Obstetrics and Gynecology, University of Washington, 1959

Pacific Street NE, Box 356460, Seattle, WA 98195, USA

Full list of author information is available at the end of the article
}

(c) The Author(s). 2018 Open Access This article is distributed under the terms of the Creative Commons Attribution 4.0 International License (http://creativecommons.org/licenses/by/4.0/), which permits unrestricted use, distribution, and reproduction in any medium, provided you give appropriate credit to the original author(s) and the source, provide a link to the Creative Commons license, and indicate if changes were made. The Creative Commons Public Domain Dedication waiver (http://creativecommons.org/publicdomain/zero/1.0/) applies to the data made available in this article, unless otherwise stated. 


\section{Background}

Magnesium sulfate $\left(\mathrm{MgSO}_{4}\right)$, the preferred pharmacological intervention for treatment of eclamptic seizures, has been shown to have greater efficacy and fewer complications than treatment with diazepam or phenytoin. [1] $\mathrm{MgSO}_{4}$ is also superior to placebo and nimodipine for prevention of eclampsia in women diagnosed with preeclampsia. [2, 3] In the MAGPIE Trial [2] the incidence of eclampsia was reduced in women with preeclampsia: RR 0.42; 95\% CI (0.29-0.60). A strong trend towards reduction in maternal mortality was also observed: RR 0.55; CI (0.26-1.14). Study subjects were treated with either an intramuscular (IM) or an intravenous (IV) regimen. The route of administration was not demonstrated to affect drug effectiveness.

Despite established efficacy, utilization of $\mathrm{MgSO}_{4}$ remains limited. Barriers to use include: the availability of $\mathrm{MgSO}_{4}$, persistent provider attitudes regarding safety and efficacy, lack of availability of infusion pumps for safe intravenous (IV) administration, and pain associated with intramuscular (IM) administration. In a randomized trial comparing IM vs. IV administration, we found that $45 \%$ of women receiving $\mathrm{IM} \mathrm{MgSO}_{4}$ considered pain associated with injections to be unacceptable or very unacceptable compared to only $2 \%$ who were treated intravenously. [4] Pain associated with $\mathrm{IM} \mathrm{MgSO}_{4}$ represents a considerable barrier to use and is potentially addressable.

We hypothesized that by administering serial intravenous (IV) boluses of $\mathrm{MgSO}_{4}$ after the loading dose instead of a continuous infusion or IM injections, we could achieve comparable serum magnesium concentrations. The loading dose and serial IV boluses could be given as a manual push by a health care provider or with a simple mechanical flow controlled intravenous pump (Springfusor ${ }^{\circ}$ ). The use of serial IV boluses would eliminate the possibility of an inadvertent overdose from a free running $\mathrm{MgSO}_{4}$ IV infusion and circumvent the need for expensive electronic infusion pumps in resource poor environments. If administered with a Springfusor ${ }^{\bullet}$ pump, a single set of flow control tubing would be required rather than one for IV bolus and a second for continuous infusion. This would reduce cost as well as eliminate the potential for inadvertent use of the incorrect flow control tubing. IV administration would significantly reduce maternal pain as a barrier to care.

Can serial IV boluses maintain sufficient serum magnesium concentrations? Abadde et al. tested administration of $\mathrm{MgSO}_{4}$ by serial IV bolus compared to continuous infusion in a randomized trial of 30 women with severe preeclampsia or eclampsia. [5] They found that serum magnesium AUC in the serial IV bolus arm was significantly lower than the AUC in the continuous infusion arm and concluded that use of the alternative serial IV bolus regimen might result in an increased risk for seizures and should not be used.
Using population PK modeling and simulations from data in our Springfusor trial, we found that maternal serum magnesium levels were lower in the IV arm compared to the IM arm. [6] After modeling the data, we suggested that use of a $6 \mathrm{~g}$ loading dose instead of a $4 \mathrm{~g}$ loading dose in the IV regimen would result in serum concentrations comparable to those achieve with the IM regimen. We subsequently evaluated the effect of a $6 \mathrm{~g}$ loading dose with a regimen using serial IV boluses through simulations and found that the expected serum concentrations would be comparable to concentrations from the IM and IV arms of the Springfusor trial. In the present study, we tested the use of a $6 \mathrm{~g}$ loading dose followed by $2 \mathrm{~g}$ IV boluses every $2 \mathrm{~h}$ in a randomized trial as compared to continuous infusion.

\section{Methods}

An open-label randomized trial was performed comparing two regimens of administering $\mathrm{MgSO}_{4}$ to women with severe preeclampsia who were deemed likely to benefit by the reduction in risk for eclamptic seizures, (trial no. NCT02091401). The trial compared maternal serum magnesium concentrations achieved by each regimen through population pharmacokinetic analysis. The study was conducted at two tertiary-care hospitals in Egypt: El Galaa Teaching Hospital in Cairo and Shatby Maternity Hospital in Alexandria. In El Galaa Hospital 16,000 births are delivered per year. The eclampsia rate is approximately $0.5 \%$. In Shatby Hospital approximately 16,000 births are delivered per year, and the rate of preeclampsia is $6.1 \%$. The study was approved by Ethics Committee of the Faculty of Medicine-Alexandria University and the El Galaa Maternity Teaching Hospital Ethical Committee. Written consent was obtained from each study participant.

To be eligible, women were required to have a systolic blood pressure $\geq 160 \mathrm{mmHg}$ or a diastolic blood pressure $\geq 110 \mathrm{mmHg}$ at two times over $30 \mathrm{~min}$ and $\geq 1$ + proteinuria. (A single higher pressure could be accepted if the clinical team felt that $\mathrm{MgSO}_{4}$ should be initiated without waiting for $30 \mathrm{~min}$.) Eligible women were pregnant or $\leq 24$ h postpartum and deemed by the admitting physician to benefit from treatment with $\mathrm{MgSO}_{4}$. Women agreed to comply with study procedures. Women were excluded if they had experienced an eclamptic seizure, had received $\mathrm{MgSO}_{4}$ within $24 \mathrm{~h}$ of study enrollment, or had a serum creatinine > $106 \mu \mathrm{mol} / \mathrm{L}(1.2 \mathrm{mg} / \mathrm{dL})$ at the time of enrollment. Subjects gave written informed consent. After informed consent, a sequentially numbered, sealed, opaque envelope containing the participant's group assignment was opened by research staff. The envelopes were generated by Gynuity Health Projects staff using a randomisation 
code based on a computerised pseudo-random number generator. Randomisation was stratified by center.

As a population PK study, the sample size $(n=100$ per group) and samples per subject $(n=7)$ were chosen to rigorously characterize the distribution and elimination of magnesium under both conditions. From this characterization, population PK modeling could then be performed. A large sample was chosen due to anticipated uncertainties regarding variability in the study population. No midterm analysis was conducted for assessment of safety.

In the serial IV bolus arm, magnesium was administered as $50 \%$ magnesium sulfate heptahydrate $\left(\mathrm{MgSO}_{4} \cdot 7 \mathrm{H} 2 \mathrm{O}\right)$ [Hospira]. In the continuous infusion arm, 10\% magnesium sulfate heptahydrate [Egyptian International Pharma ceutical Industries Company]was used, the community standard. Women were randomized to receive one of two regimens. The CONTINUOUS INFUSION ARM was the community standard at each hospital. A $4 \mathrm{~g}$ intravenous loading dose of $10 \% \mathrm{MgSO}_{4}$ was administered manually over approximately $20 \mathrm{~min}$ as done in the MAGPIE Trial. [2] At the conclusion of the loading dose a continuous infusion of $\mathrm{MgSO}_{4}$ at $1 \mathrm{~g}$ /hour by mini-drip was started (the community standard). In the SERIAL IV BOLUS ARM, $50 \% \mathrm{MgSO}_{4}$ was administered by Springfusor ${ }^{\circ}$ springloaded pump, (GoMedical, Subiaco, Australia), through a flow control tubing designed to deliver $10 \mathrm{~mL}$ of saline over $5 \mathrm{~min}$ and calibrated to deliver the viscous $50 \%$ $\mathrm{MgSO}_{4}$ solution to study requirements. A $6 \mathrm{~g}(12 \mathrm{~mL})$ intravenous loading dose was administered over approximately $30 \mathrm{~min}$. Subsequently, a $2 \mathrm{~g}(4 \mathrm{~mL})$ IV bolus was administered over $10 \mathrm{~min}$ every $2 \mathrm{~h}$. Clinical care was managed by local standards. The loading dose and subsequent IV boluses were delivered through a side port of an IV infusion running at $100-150 \mathrm{ml}$ per hour to achieve adequate dilution of the $50 \%$ solution. The delivery of $50 \%$ solution was only $4 \mathrm{ml}$ per minute.

Maternal serum samples were collected over a 12-h infusion period.

Each subject had a baseline and 6 strategically timed blood samples drawn during the study period for measurement of magnesium concentrations. The samples were distributed to be informative for a population pharmacokinetic (PK) analysis. Sample collection times are in relation to the end of the loading dose. Subjects in the continuous infusion arm were assigned to one of three groups for sample collection times. Continuous Infusion Group-1 $(n=33)$ collected samples at time Baseline, 20, 80, 160, 280, 500 and $740 \mathrm{~min}$. Continuous Infusion Group-2 $(\mathrm{n}=33)$ collected samples at time Baseline, 60, 120, 240, 420, 580 and 740 min. Continuous Infusion Group-3 $(n=34)$ collected samples at time Baseline, 40, 100, 200, 340, 660 and 740 min. Subjects in the serial IV bolus arm were also assigned to one of three groups for sample collection times. Serial Bolus Group-1 $(\mathrm{n}=33)$ collected samples at time Baseline, 20, 100, 200, 380, 660 and $750 \mathrm{~min}$. Serial Bolus Group-2 ( $\mathrm{n}=$ 33) collected samples at time Baseline, 40, 140, 300, 400, 500 and $600 \mathrm{~min}$. Serial Bolus Group-3 $(\mathrm{n}=34)$ collected samples at time Baseline, 60, 160, 260, 340, 440 and $620 \mathrm{~min}$. Actual drug administration and sampling times were used in the analysis. A serum creatinine level was drawn at baseline and at the conclusion of the study period. At Shatby hospital, magnesium levels were measured in the hospital laboratory with a Cobass 501 system using a colorimetric endpoint method (Roche Diagnostics). At El Galaa Hospital, magnesium levels were measured in the hospital laboratory with BT-3500 autoanalyzer using colorometric endpoint method, (Biotecnia Instruments $\operatorname{SpA}$ ).

At the conclusion of treatment with $\mathrm{MgSO}_{4}$, subjects were interviewed and asked to grade side effects as mild, moderate or severe. They were also asked to rate the overall acceptability of side effects as "very acceptable," "acceptable," "neutral," "unacceptable" or "very unacceptable". (This approach, while used extensively, has not been validated for reproducibility in our hands).

Serum magnesium concentrations were analyzed with nonlinear mixed effect model using NONMEM (version 7.3.0). [7] One and two-compartmental models with first order elimination were evaluated for goodness-of-fit diagnostics. Maximum likelihood population parameter estimates were determined for clearance $(\mathrm{CL})$, volume of distribution (V) and baseline endogenous steady-state magnesium concentration (BL). The administered magnesium was modeled as additive to BL. Combined data as well as continuous infusion data and short infusion data were modeled for goodness of fit. Combined data was used for the final model.

After determination of the base model, maternal age, actual body weight, ideal body weight, height, gestational age, serum creatinine at the start of the PK study, serum creatinine at the end of the PK study, creatinine clearance at the start of the PK study, creatinine clearance at the end of the PK study and number of fetuses were tested as linear, additive, exponential, proportional and power model covariates as appropriate for potential inclusion in the final model. Creatinine clearance was determined using the Cockcroft and Gault equation. [8] Weight adjustments were normalized for the mean body weight in the study $(90.54 \mathrm{~kg})$. The final model inclusion of a covariate was based on statistical significance with a decrease in objective function of at least $>3.84(P<0.05)$.

Following determination of the final model, Monte Carlo simulation was used to generate a continuous infusion population with 200 subjects and a serial IV bolus population with 200 subjects receiving the dosing for the original study design as described above. The simulations utilized the final population model parameter 
estimates of fixed effects as well as inter-individual and residual random variability. Body weight was varied within a range of $\pm 30 \%$ of the mean body weight. The $12 \mathrm{~h}$ and $20 \mathrm{~min}$ area under the concentration-time curve was estimated for each group using linear trapezoidal rule.

The study was funded by Merck for Mothers who had no role in study design, data collection, data analysis, data interpretation, or writing of the report. The corresponding author had full access to all data in the study and had final responsibility for the decision to submit for publication.

\section{Results}

Two hundred women were enrolled in the study, 100 from El Galaa Teaching Hospital in Cairo and 100 from Shatby Maternity Hospital in Alexandria between January 2015February 2016. The Consort Diagram is displayed in Fig. 1.
Table 1 describes maternal characteristics. Only the incidence of primigravidity appeared different between groups. Table 2 describes maternal outcomes that were similar between groups. No adverse maternal outcomes such as eclampsia, maternal death, or the need for transfusion were experienced. In the serial IV bolus arm, 8 women stopped treatment early due to side effects $(n=4)$, signs of toxicity $(n=2)$, fetal distress $(n=1)$ or provider error $(n=1)$. In cases where treatment was stopped early due to signs of toxicity (bradycardia, decreased respiratory rate, or absent reflexes), calcium gluconate was administered, women recovered fully and no ICU admission was required. In the continuous infusion arm, 6 women stopped treatment early due to delivery $(n=3)$, low urinary output $(n=2)$ or provider request $(n=1)$.

Table 3 describes neonatal outcomes that were similar between groups. Complications were largely those related to preterm birth.

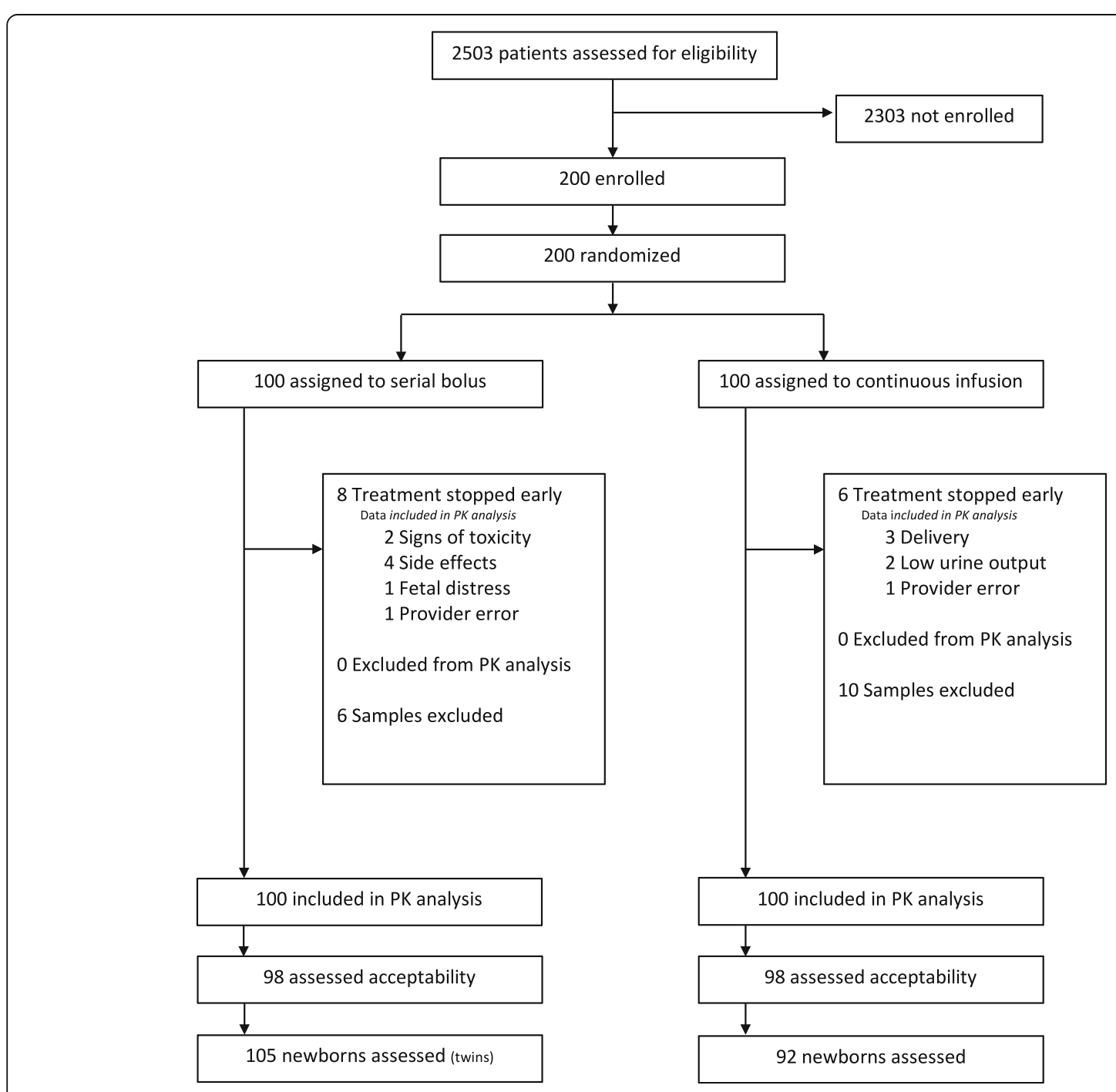

Fig. 1 Consort Diagram 
Table 1 Maternal Characteristics

\begin{tabular}{|c|c|c|c|c|}
\hline \multicolumn{2}{|c|}{ 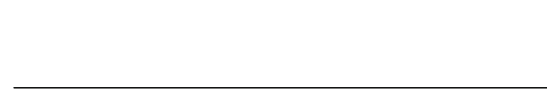 } & & $\frac{\text { Serial IV Bolus }}{(n=100)}$ & $\frac{\text { Continuous Infusion }}{(n=100)}$ \\
\hline \multicolumn{3}{|c|}{ Maternal age (years) mean \pm SD } & $29 \pm 6$ & $29 \pm 6$ \\
\hline \multicolumn{3}{|c|}{ Weight $(\mathrm{kg})$ mean $\pm \mathrm{SD}$} & $91.4 \pm 17.2$ & $89.6 \pm 14.8$ \\
\hline \multicolumn{3}{|c|}{ Gestational Age (weeks) mean \pm SD } & $35.7 \pm 2.8$ & $35.2 \pm 3.3$ \\
\hline \multicolumn{3}{|l|}{ Primigravid - N (\%) } & $46(46 \%)$ & $24(24 \%)$ \\
\hline \multicolumn{3}{|c|}{ Multiple Pregnancy - N (\%) } & $5(5 \%)$ & $4(4 \%)$ \\
\hline \multicolumn{3}{|c|}{ Postpartum enrollment- N (\%) } & $0(0 \%)$ & $2(2 \%)$ \\
\hline \multicolumn{3}{|c|}{ Systolic BP $(\mathrm{mmHg})$ mean $\pm \mathrm{SD}$} & $167 \pm 10.9$ & $165 \pm 7.7$ \\
\hline \multicolumn{3}{|c|}{ Diastolic BP $(\mathrm{mmHg})$ mean \pm SD } & $113 \pm 7.7$ & $113 \pm 5.9$ \\
\hline \multirow[t]{3}{*}{ Proteinuria - N (\%) } & $<1+$ & & 0 & 0 \\
\hline & $1+$ & & $10(10 \%)$ & $17(17 \%)$ \\
\hline & $\geq 2+$ & & $90(90 \%)$ & $83(83 \%)$ \\
\hline \multicolumn{5}{|l|}{ Serum creatinine } \\
\hline \multirow[t]{4}{*}{ Initial } & $(\mu \mathrm{mol} / \mathrm{L})$ & mean $\pm \mathrm{SD}$ & $66.3 \pm 15.9$ & $66.3 \pm 15.9$ \\
\hline & & range & $26.5-106$ & $26.5-106$ \\
\hline & $(\mathrm{mg} / \mathrm{dL})$ & mean $\pm \mathrm{SD}$ & $0.75 \pm 0.18$ & $0.75 \pm 0.18$ \\
\hline & & range & $0.3-1.2$ & $0.3-1.2$ \\
\hline \multirow[t]{4}{*}{ Final } & $(\mu \mathrm{mol} / \mathrm{L})$ & mean $\pm S D$ & $73.4 \pm 15.9$ & $74.3 \pm 16.8$ \\
\hline & & range & $44.2-133$ & $26.5-141$ \\
\hline & $(\mathrm{mg} / \mathrm{dL})$ & mean $\pm \mathrm{SD}$ & $0.83 \pm 0.18$ & $0.84 \pm 0.19$ \\
\hline & & range & $0.50-1.5$ & $0.30-1.6$ \\
\hline
\end{tabular}

From the 200 study subjects, 1347 magnesium concentrations were obtained. Of these, 16 were excluded from analysis for one of two reasons: 1) the concentrations appeared to be contaminated by the magnesium infusion or 2) the concentration at time zero was higher than all other concentrations during the study suggesting the sample was mislabeled or contaminated by the infusion.

A one-compartment linear elimination model provided the best fit of the data. Using weight (exponential) as a covariate for volume and serum creatinine at the start of the study (linear) as a covariate for clearance provided the greatest decrease in the objective function and were utilized in the final model. Fig. 2A depicts a scatterplot of observed magnesium concentrations versus model-population predicted magnesium concentrations for the final model. Fig. 2B depicts a scatterplot of the observed magnesium concentrations versus model-individually predicted magnesium concentrations. Fig. 2C depicts weighted residuals versus

Table 2 Maternal Outcomes

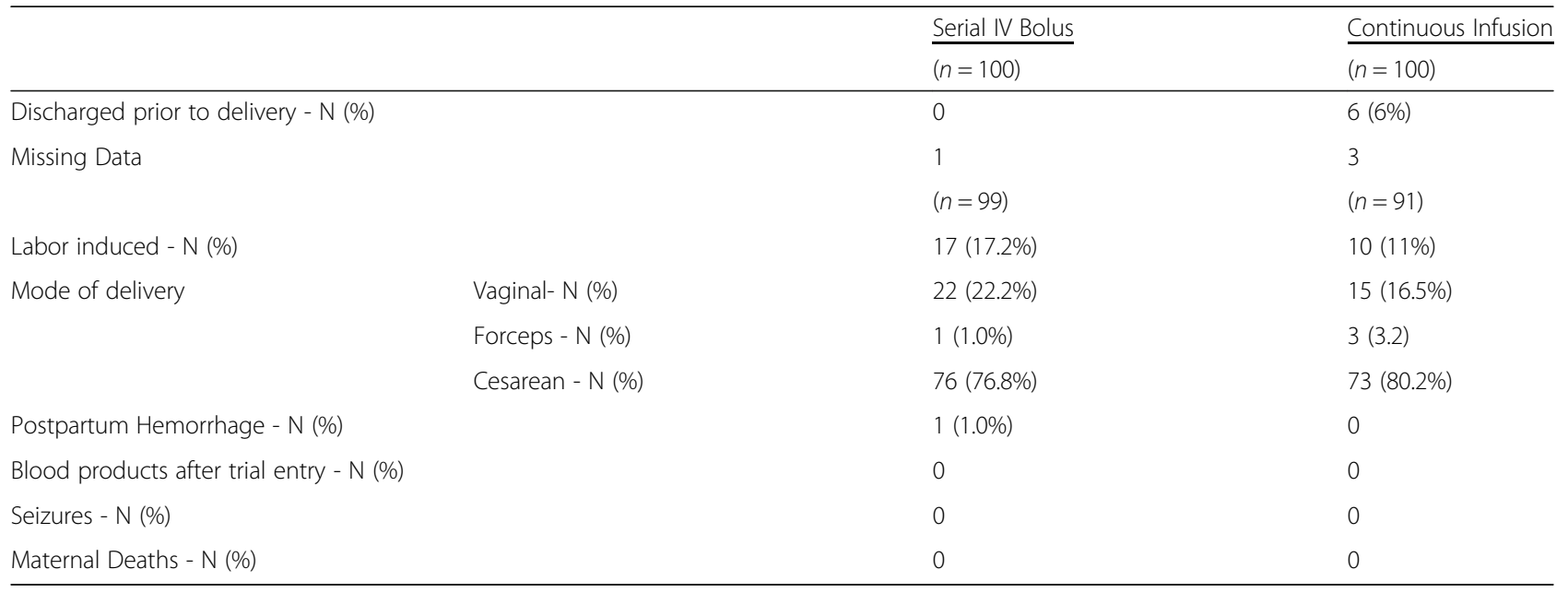


Table 3 Neonatal Outcomes

\begin{tabular}{lll}
\hline & Serial IV Bolus & Continuous Infusion \\
\cline { 2 - 3 } Mothers enrolled & $n=100$ & $n=100$ \\
Enrolled postpartum & 0 & 2 \\
Discharged prior to delivery & 0 & 6 \\
Twin Infants & 6 & 2 \\
Missing data & 1 & 2 \\
Newborns evaluated & $n=105$ & $n=92$ \\
Live Birth- N (\%) & $105(100 \%)$ & $91(98.9 \%)$ \\
Stillbirth- N (\%) & 0 & $1(1.1 \%)$ \\
Intubated at delivery - N (\%) & $6(5.7 \%)$ & $6(6.5 \%)$ \\
Mechanical Ventilation - N (\%) & $3(2.8 \%)$ & $6(6.5 \%)$ \\
Bradycardia < 110/min - N (\%) & $2(1.9 \%)$ & $4(4.3 \%)$ \\
Special Care Nursery - N (\%) & $25(23.8 \%)$ & $26(28.2 \%)$ \\
Neonatal Deaths - N (\%) & $2(1.9 \%)$ & $3(3.2 \%)$ \\
Prematurity & 0 & 1 \\
Unknown & 2 & 2 \\
\hline
\end{tabular}

model predicted concentrations demonstrating a fairly symmetric distribution. Comparing the diagnostic plots for base and final models demonstrate that the inclusion of weight as a covariate for volume and serum creatinine as a covariate for clearance substantially improves the prediction of magnesium concentrations. However, unexplained clinical variability in magnesium concentrations was still apparent (data not shown).

Pharmacokinetic parameters estimated from the continuous infusion subjects alone and the serial IV bolus subjects alone were comparable to parameters estimated using all data combined (data not shown), therefore all data combined was used in the final model. Table 4 reports the estimated average clearance, volume of distribution and baseline magnesium concentration from the population analysis. Also reported are the effects of weight on magnesium volume and serum creatinine at the start of the study on magnesium clearance.

Figure 3 depicts the actual individual magnesium concentrations plotted vs. time where time zero is at the initiation of the loading dose. Blue dots represent the subjects receiving serial IV boluses and red dots represent the subjects receiving a continuous infusion. (This graph excludes one subject that had a deviation from study protocol that resulted in samples obtained well beyond the end of the study period. Data was included in the PK analysis). The graphs demonstrate a broad overlap in magnesium concentrations in the two treatment arms.

Figure 4 demonstrates the appropriateness of the model in describing the data. Figure 4A depicts the median simulated magnesium concentration-time profile for the continuous infusion arm (black line) and the observed magnesium concentrations (red dots). Fig. 4B

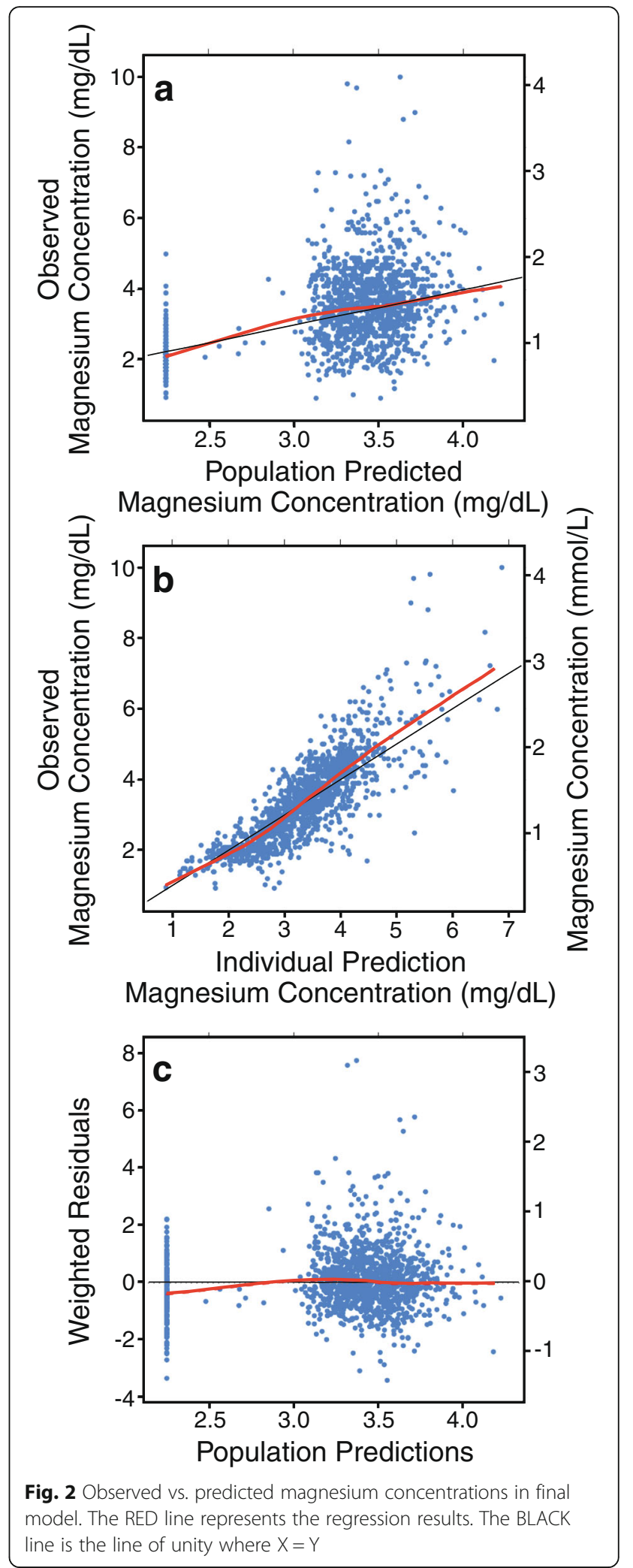


Table 4 Population pharmacokinetic model parameters mean \pm SE

\begin{tabular}{|c|c|c|}
\hline \multicolumn{2}{|l|}{ Clearance (mL/min) } & $56.3 \pm 12.7$ \\
\hline \multicolumn{2}{|l|}{ Volume of distribution (L) } & $65.3 \pm 13.1$ \\
\hline \multirow[t]{2}{*}{ Baseline Mg concentration } & $(\mathrm{mmol} / \mathrm{L})$ & $0.925 \pm 0.037$ \\
\hline & $(\mathrm{mg} / \mathrm{dL})$ & $2.25 \pm 0.09$ \\
\hline \multicolumn{2}{|c|}{ Weight effect on volume exponential $\left(\theta_{1}\right)$} & $-0.426 \pm 0.196$ \\
\hline \multicolumn{2}{|c|}{ Serum creatinine effect on exponential $\left(\theta_{1}\right)$} & $0.553 \pm 0.138$ \\
\hline \multicolumn{2}{|l|}{ Residual variability } & $0.0387 \pm 0.0013$ \\
\hline
\end{tabular}

depicts the median simulated magnesium concentrationtime profile for the serial IV bolus arm (black line) and the observed magnesium concentrations (blue dots).

Figure 5 depicts the median magnesium concentration time-curves for the continuous infusion simulated population (red line, $n=200$ ) and the serial IV bolus simulated population (blue line, $n=200)$. The average $( \pm S D)$ simulated $12 \mathrm{~h}$ and 20 min magnesium AUC was 1010 $\pm 398 \mathrm{mmol} \bullet \mathrm{min} / \mathrm{L},(2458 \pm 969 \mathrm{mg} \bullet \mathrm{min} / \mathrm{dL})$, for the continuous infusion arm and $1107 \pm 461 \mathrm{mmol} \bullet \mathrm{min} / \mathrm{L}$, $(2694 \pm 1123 \mathrm{mg} \bullet \mathrm{min} / \mathrm{dL})$, for the serial IV bolus arm $(P=0.02)$. Mean magnesium serum concentration was derived by dividing the AUC by the total time. Mean concentration in the serial IV bolus arm was $1.49 \mathrm{mmol} /$ $\mathrm{L}(3.62 \mathrm{mg} / \mathrm{dL})$ compared to $1.36 \mathrm{mmol} / \mathrm{L}(3.32 \mathrm{mg} / \mathrm{dL})$ in the continuous infusion arm.

Table 5 describes maternal side effects and acceptability of treatment associated with each treatment arm. Women receiving serial IV boluses reported more mild to moderate pain, largely at the infusion site during boluses, $(P<0.05)$. There was also a trend towards more flushing in the serial IV bolus group. Only a small number of subjects in each group rated the treatment as unacceptable or very unacceptable.

\section{Discussion}

We found that magnesium sulfate administered by serial IV boluses achieved serum magnesium concentrations significantly higher but clinically equivalent to those achieved with a continuous infusion offering a third option for treatment of women with preeclampsia. Magnesium sulfate is the preferred treatment of eclamptic seizures and for the prevention of seizures in women with preeclampsia. Our goal was to reduce barriers to treatment with $\mathrm{MgSO}_{4}$ by developing an alternative regimen that would avoid the pain and lack of acceptability of intramuscular injections and the need for an electronic pump to safely regulate an intravenous infusion. A clinical trial based on the incidence of seizures was not deemed feasible. To examine a potential $50 \%$ increase in the incidence of eclampsia from $0.8 \%$ reported in the $\mathrm{MgSO}_{4}$ arm of the MAGPIE Trial to $1.2 \%$ would require a sample size of more than 9000 subjects in each arm. We therefore chose to test pharmacological equivalence by comparing the serum AUC achieved by each regimen.

We found that a one-compartment linear elimination model provided the best fit of the data, similar to prior studies. [6, 9] Body weight and serum creatinine improved model fit as previously described. [6] Our estimated clearance, $56.3 \mathrm{~mL} / \mathrm{min}$, was similar to those previously reported by Brookfield et al., $(66.3 \mathrm{~mL} / \mathrm{min})$, Salinger et al., $(80.1 \mathrm{~mL} / \mathrm{min})$, and Chuan et al., $(71.3 \mathrm{~mL} / \mathrm{min})[6,9,10]$. However, our volume of distribution, $(65.5 \mathrm{~L})$, and body weight, $(90.5 \mathrm{~kg})$, were higher

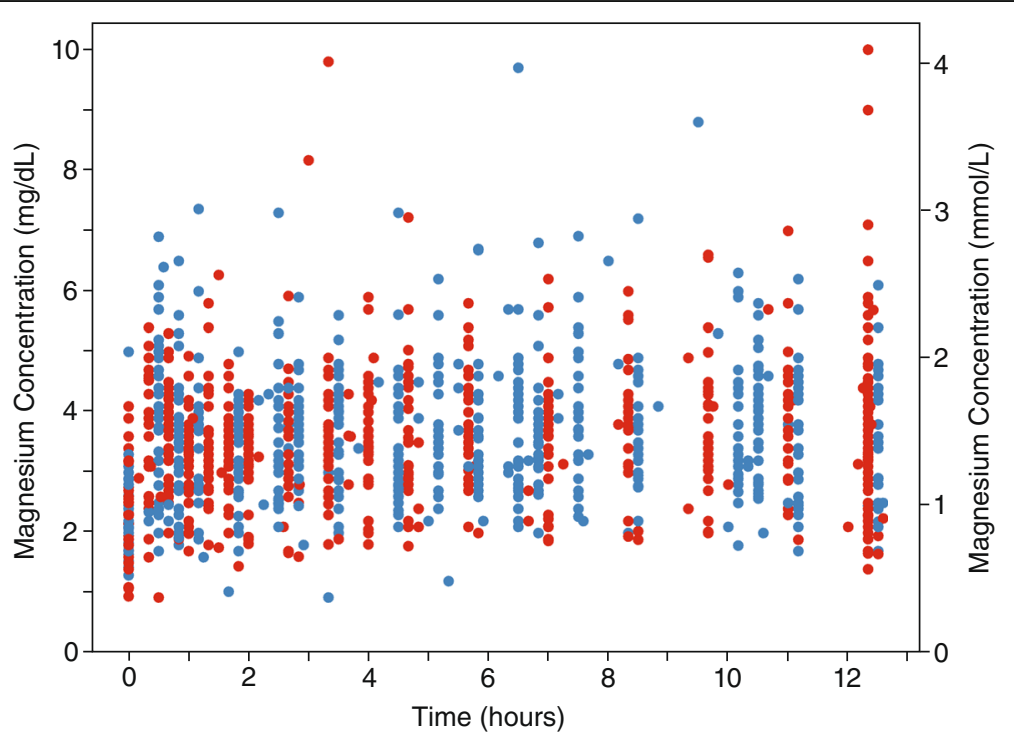

Fig. 3 Individual magnesium concentrations over time. A-Continuous Infusion. B-Serial IV Bolus 

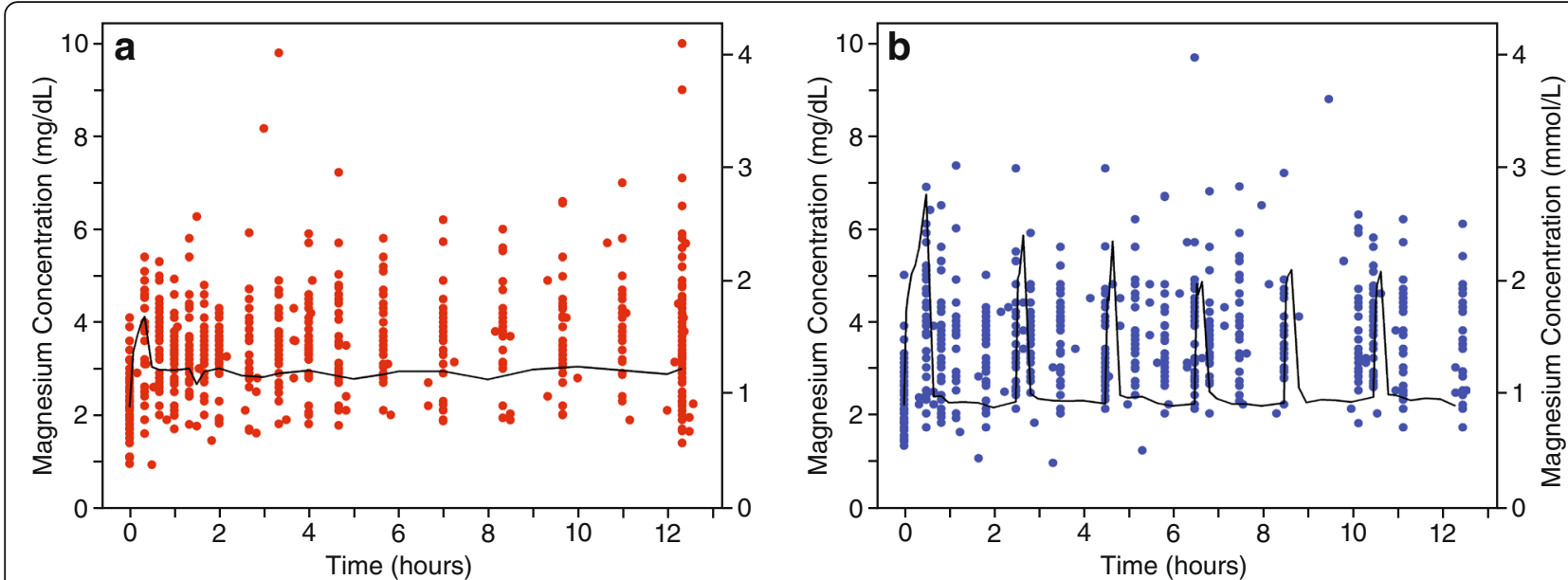

Fig. 4 Median simulated magnesium concentrations over time with observed magnesium concentrations. A-Continuous Infusion. B-Serial IV Bolus

than previously reported by Brookfield et al. (22.5 L; $87.5 \mathrm{~kg})$, Salinger et al. (15.6 L; $57.3 \mathrm{~kg})$, Lu et al. $(49 \mathrm{~L} ; 68 \mathrm{~kg})$ and Chuan et al. $(32.3 \mathrm{~L} ; 65 \mathrm{~kg}$, pre-pregnancy)[6, 9-11].

The degree of variability described in Fig. 3 demonstrates a fundamental challenge to dosing $\mathrm{MgSO}_{4}$ empirically without reference to individual serum magnesium concentrations. Nevertheless, this is the only feasible approach in low resource settings. We have previously reported a similar variability in a cohort of Indian women. [6] This residual variability in the model unexplained by maternal weight or renal function is likely due to differences in volume of distribution associated with differences in extracellular fluid including plasma volume and edema. Any difference in magnesium exposure associated with different regimens must be interpreted in

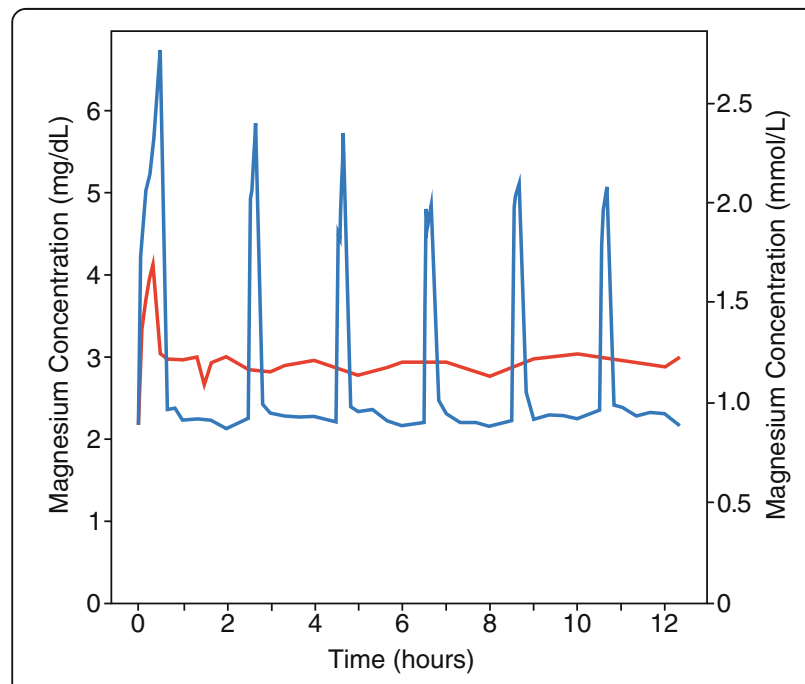

Fig. 5 Median simulated magnesium concentrations over time. RedContinuous Infusion. Blue-Serial IV Bolus context of the background variability. The observed variability poses an inherent challenge in achieving appropriate dosing - neither too low nor too high - with a uniform dosing strategy.

The total magnesium exposure, as measured by AUC, using simulated populations was higher in the serial IV bolus group, (Fig. 5). Higher peak concentrations associated with serial IV boluses were offset by lower trough concentrations. Although the AUC was significantly higher in the serial IV bolus arm, the mean differences are probably not clinically significant in the context of observed variability. Does the higher AUC in the serial IV bolus arm represent equivalent or superior efficacy despite lower trough concentrations? Given a lack of understanding of the mechanism of seizure prevention associated with magnesium, this question is difficult to answer but worthy of consideration. If the mechanism of action is due to vasomotor relaxation exhibited by patient flushing, serial IV boluses could be more effective. If the site of action is outside the blood stream, the high serum levels during IV boluses would drive a more rapid redistribution into the tissue and might be more effective. Alternatively, if efficacy is dependent on steady state serum concentration, the serial IV bolus regimen might be less effective. As previously discussed, the magnitude of variability between subjects probably overshadows these arguments.

The study was not powered to detect differences in maternal and neonatal outcomes. We did not find any suggestion of worse outcomes for mothers receiving serial IV boluses. Although serial IV boluses resulted in a higher AUC, the magnitude of these differences was small in the context of the variability in serum level. The observed high serum levels were, on inspection, distributed comparably across study groups. $\mathrm{MgSO}_{4}$ infusions were stopped early in $8 \%$ of women in the serial IV 
Table 5 Side Effects

\begin{tabular}{|c|c|c|}
\hline & $\underline{\text { Serial IV Bolus }}$ & Continuous Infusion \\
\hline & $n=98$ & $n=98$ \\
\hline Nausea- N (\%) & $10(10.2 \%)$ & $8(8.2 \%)$ \\
\hline Mild & 6 & 6 \\
\hline Moderate & 3 & 2 \\
\hline Severe & 1 & 0 \\
\hline Vomiting - N (\%) & $5(5.1 \%)$ & $2(2.0 \%)$ \\
\hline Mild & 1 & 0 \\
\hline Moderate & 3 & 2 \\
\hline Severe & 1 & 0 \\
\hline Flushing - N (\%) & $18(18.4 \%)$ & $9(9.2 \%)$ \\
\hline Mild & 16 & 9 \\
\hline Moderate & 0 & 0 \\
\hline Severe & 2 & 0 \\
\hline Drowsiness - N (\%) & $15(15.3 \%)$ & $13(13.2 \%)$ \\
\hline Mild & 11 & 7 \\
\hline Moderate & 3 & 6 \\
\hline Severe & 1 & 0 \\
\hline Pain - N (\%) & $28(28.6 \%)$ & $9(9.2 \%)(P<0.05)$ \\
\hline Mild & 20 & 9 \\
\hline Moderate & 7 & 0 \\
\hline Severe & 1 & 0 \\
\hline Acceptable/Very acceptable & $88(89.8 \%)$ & $94(95.9 \%)$ \\
\hline Neutral & $6(6.1 \%)$ & $2(2.0 \%)$ \\
\hline Unacceptable/Very unacceptable & $4(4.0 \%)$ & $2(2.0 \%)$ \\
\hline
\end{tabular}

bolus arm compared to $6 \%$ in the continuous infusion arm. This compares to a discontinuation rate of $16 \%$ in the treatment arm of the MAGPIE Trial. [2] The incidence of neonates requiring mechanical ventilation in the serial IV bolus arm, (2.8\%) was lower than that in the continuous infusion arm (6.5\%). The incidence of bradycardia in the serial IV bolus arm (1.9\%) was lower than that in the continuous infusion arm (4.3\%). These results suggest that an increased incidence of adverse outcomes using serial IV boluses is unlikely.

The Springfusor pump was used to achieve a standardized infusion rate in the serial IV bolus arm. The flow-control tubing effectively prevents an accidental rapid infusion and does not require constant staff attention. Serial IV boluses could be performed manually at the same rate by a health care provider in the absence of a Springfusor pump but would be subject to variability in infusion rate and would require greater use of provider resources. The serial IV bolus approach could be adapted to the concentration of $\mathrm{MgSO}_{4}$ used at a specific site and to the local method used to administer an IV loading dose. Local conditions, balancing the cost and availability of health care staff against the cost and availability of the Springfusor ${ }^{\circledR}$ pump system, would drive local decision making.

Few women in either arm found the treatment unacceptable or very unacceptable though women in the serial IV bolus reported more pain, generally mild to moderate. Pain was at the infusion site. Unacceptable pain or very unacceptable pain $(4.0 \%)$ was much less than we have reported with use of IM MgSO4 (29.4\%). [4] While inclusion of lidocaine with IM MgSO4 may improve acceptability above that seen in our prior trial, it is unlikely to impact pain of the injection itself. There was also a tendency to report more flushing. By decreasing the infusion rate with a slower flow control tubing, we might be able to decrease pain at the infusion site and flushing. This strategy would also decrease peak concentrations associated with each IV bolus without affecting the total magnesium exposure. Increasing infusion rate of the back-up IV will also serve to decrease pain with infusion.

This study was conducted in lower resource settings, the intended site of potential use, which could pose inherent challenges in performing a pharmacokinetic study. Some individual serum samples were excluded due to what appeared to be protocol violations and may have been due to a lapse in appropriate attention to blood draws at the beginning of the protocol. This affected only $1.1 \%$ of total samples.

We have demonstrated that serial IV boluses represent a third option for the administration of $\mathrm{MgSO}_{4}$ to women with preeclampsia. Serial IV boluses achieve serum magnesium concentrations significantly higher but clinically equivalent to those achieved with a continuous infusion. This strategy avoids barriers to care associated with painful IM injections and the need for an electronic continuous infusion pump, not readily available in low resource settings. It can also be initiated prior to transport of a patient to a higher level of care without the need to treat during transport. The use of a Springfusor ${ }^{\oplus}$ pump, while fundamentally not required, insures the consistency of infusion rate and prevents an overly rapid manual infusion if administered by a provider.

\section{Conclusions}

Serial IV boluses can be used to administer $\mathrm{MgSO}_{4}$ to pregnant women at risk for eclampsia. Adoption of this strategy may overcome barriers associated with IM injections and continuous infusions.

\section{Abbreviations}

AUC: area under the curve; BL: baseline; CL: clearance; dL: deciliter; g: gram hr.: hour; IM: intramuscular; IV: intravenous; $\mathrm{MgSO}_{4}$ : magnesium sulfate; MgSO4.7H20: magnesium sulfate heptahydrate; min: minute; $\mathrm{mL}$ : milliliter; mmHg: millimeters of mercury; mmol: millimole; PK: pharmacokinetic;

V: volume of distribution; $\mu$ mol: micromole 


\section{Acknowledgements}

Dr. Sherif Mohamed Safwal, Dr. Ahmed Shokry Nasr and Dr. Tamer AbdelAziz assisted with study coordination at the study sites. Miral Breebaart and Roxanne Martin assisted with managing, cleaning, and entering data.

\section{Funding}

The study was funded by Merck for Mothers who had no role in study design, data collection, data analysis, data interpretation, or writing of the report.

\section{Availability of data and materials}

The datasets used and/or analysed during the current study are available from the corresponding author on reasonable request.

\section{Authors' contributions}

TE collaborated on study design, participated in initiation of the study in Egypt, and wrote the first draft. MH performed the population PK analysis, assisted in drafting the manuscript, and reviewed and contributed to the final manuscript. HB collaborated on study design, participated in initiation of the study in Egypt, supervised study in Egypt, assisted in drafting the manuscript, and reviewed and contributed to the final manuscript. ED participated in the design of the study to meet local conditions, initiation of the study in Egypt, directed the study on the ground in Egypt and reviewed the final manuscript. MR participated in the design of the study to meet local conditions, initiation of the study in Egypt, directed the study on the ground in Egypt and reviewed and contributed to the final manuscript. SS participated in the design of the study to meet local conditions, initiation of the study in Egypt, directed the study on the ground in Egypt and reviewed and contributed to the final manuscript. DC participated in the design of the study to meet local conditions, initiation of the study in Egypt, monitored study progress on the ground in Egypt and reviewed and contributed to the final manuscript. TA coordinated study enrollment and data review and quality control at Shatby hospital and reviewed and contributed to the final manuscript. AN coordinated study enrollment and data review and quality control at El Galaa hospital and reviewed and contributed to the final manuscript. SMS coordinated study enrollment and data review and quality control at El Galaa hospital and reviewed and contributed to the final manuscript. BW collaborated on study design and reviewed and contributed to the final manuscript. Each author participated sufficiently in the work to take public responsibility for appropriate portions of the content, agreed to be accountable for all aspects of the work in ensuring that questions related to the accuracy or integrity of any part of the work are appropriately investigated and resolved, and read and approved the final manuscript.

\section{Ethics approval and consent to participate}

The study was approved by Ethics Committee of the Faculty of Medicine-Alexandria University and the El Galaa Maternity Teaching Hospital Ethical Committee. Written consent was obtained from each subject.

\section{Consent for publication}

Not applicable.

\section{Competing interests}

The authors declare that they have no competing interests.

\section{Publisher's Note}

Springer Nature remains neutral with regard to jurisdictional claims in published maps and institutional affiliations.

\section{Author details}

${ }^{1}$ Department of Obstetrics and Gynecology, University of Washington, 1959 Pacific Street NE, Box 356460, Seattle, WA 98195, USA. ${ }^{2}$ School of Pharmacy, University of Washington, Seattle, WA, USA. ${ }^{3}$ Gynuity Health Projects, New York, NY, USA. ${ }^{4}$ Shatby Maternity Hospital, Alexandria University, Alexandria, Egypt. ${ }^{5}$ El Galaa Teaching Hospital, Cairo, Egypt.
Received: 2 June 2017 Accepted: 26 June 2018

Published online: 06 July 2018

\section{References}

1. The Eclampsia Trial Collaborative Group. Which anticonvulsant for women with eclampsia? Evidence from the collaborative Eclampsia trial. Lancet. 1995;345:1455-63.

2. The Magpie Trial Collaboration Group. Do women with pre-eclampsia, and their babies, benefit from magnesium sulphate? The Magpie Trial: a randomised placebo-controlled trial. Lancet. 2002;359:1877-90.

3. Belfort M, Anthony J, Saade G, Allen J. A comparison of magnesium sulfate and nimodipine for the prevention of eclampsia. $N$ Engl J Med. 2003:348:304-11.

4. Mundle S, Regi A, Easterling T, Biswas B, Bracken H, Khedekar V, et al. Treatment approaches for preeclampsia in low-resource settings: a randomized trial of the Springfusor pump for delivery of magnesium sulfate. Pregnancy Hypertension. 2012:2:32-8.

5. Abbade J, Costa R, Martins A, Borges V, Rudge M, Peraçoli J. Zuspan's scheme versus an alternative magnesium sulfate scheme: randomized clinical trial of magnesium serum concentrations. Hypertension in Pregnancy. 2010;29:82-92.

6. Salinger D, Mundle $S$, Regi A, Bracken $H$, Winikoff $B$, Vicini $P$, et al. Magnesium sulfate for prevention of eclampsia: are intramuscular and intravenous regimens equivalent? A population pharmacokinetic study. BJOG. 2013;120:894-900.

7. Beal S, Sheiner LB, Boeckmann A, Bauer RJ. NONMEM User's Guides. Ellicott City, MD, USA: Icon development solutions; 2009.

8. Cockcroft D, Gault M. Prediction of creatinine clearance from serum creatinine. Nephron. 1976;16:31-41.

9. Chuan F, Charles B, Boyle R, Rasiah R. Population pharmacokinetics of magnesium in preeclampsia. Am J Obstet Gynecol. 2001;185:593-9.

10. Brookfield K, Su F, Elkomy M, Drover D, Lyell D, Carvalho B. Am J Obstet Gynecol. 2016:214:737-9.

11. Lu J, Pfister M, Ferrari P, Chen G, Sheiner L. Pharmacokinetic-pharmacodynamic modelling of magnesium plasma concentration and blood pressure in preeclamptic women. Clin Pharmacokinet. 2002;41:1105-13.

\section{Ready to submit your research? Choose BMC and benefit from:}

- fast, convenient online submission

- thorough peer review by experienced researchers in your field

- rapid publication on acceptance

- support for research data, including large and complex data types

- gold Open Access which fosters wider collaboration and increased citations

- maximum visibility for your research: over $100 \mathrm{M}$ website views per year

At BMC, research is always in progress.

Learn more biomedcentral.com/submissions 